\title{
Über die Impressoriensammlung im Wiener Staatsarchiv als Quelle der Wissenschaftsgeschichte
}

\author{
Jürgen Hamel \\ Archenhold-Sternwarte \\ Alt-Treptow 1, D-12435 Berlin, Germany
}

\begin{abstract}
An overview on various privileges concerning imperial printing rights (early forms of copyright) in the Austrian Staatsarchiv allows to trace the creation of several predominant astronomical publications. This is demonstrated by three examples: Jost Bürgi, Erasmus Reinbold and Christoph Rothmann.
\end{abstract}

Zusammenfassung:

Im Österreichischen Staatsarchiv in Wien befindet sich eine umfangreiche Sammlung kaiserlicher Privilegien für die Herausgabe von Büchern. Die Analyse dieser Privilegien, eine Vorform unseres Copyrights, vermag Einsichten in die Entstehungsgeschichte einzelner Werke sowie die Lebensgeschichte ihrer Autoren zu vermitteln. Dies wird anhand von drei Beispielen verdeutlicht.

Mein Anliegen ist es, mit den Beispielen von Jost Bürgi, Erasmus Reinhold und Christoph Rothmann auf eine Quelle der wissenschaftsgeschichtlichen Forschung aufmerksam zu machen, die bislang noch viel zu wenig genutzt wurde: die Impressoriensammlung im Österreichischen Staatsarchiv (Haus-, Hof- und Staatsarchiv) in Wien. Worum geht es? Bekannt ist, daß sich in der Vergangenheit Buchautoren und Hersteller von Instrumenten ein Kaiserliches Privileg erteilen ließen, das für einen unterschiedlichen Zeitraum gewährt wurde, für 3, 6, 10 Jahre und mehr. Auf dem Titelblatt wurde dies vermerkt mit einem Wortlaut, wie "Mit Kaiserlicher Freiheit nit nachzudrucken" und entsprechenden lateinischen Vermerken, wie "Cum gratia \& privilegio Caesareae \& Regiae Maiestatis", um an dieser Stelle eine Langfassung anzuführen.

Die genannte Wiener Sammlung umfaßt Hunderte von Privilegien aus der Zeit von etwa 1550 bis um 1800, daneben betreffende Antragsschreiben und gelegentlich Stellungnahmen und Ergänzungen von verschiedener Seite. 
Diese Art der Privilegien stellte einen Vorläufer des Copyrights und des Patentschutzes dar und wie es heute um Verstöße gegen diese "Privilegien" Gerichtsprozesse gibt, fanden sie gelegentlich auch damals statt.

Ohne weiter in die Theorie und die Rechtsformen der alten Privilegien einzugehen ${ }^{1}$, möchte ich nur anführen, daß es sowohl Autorenprivilegien gab, und hier wieder sowohl für ein einzelnes Werk, als auch für eine Gesamtheit geplanter Werke, ebenso Druckprivilegien und Editionsprivilegien, d.h. Privilegien für Drucker bzw. für Körperschaften zur Herausgabe von Büchern. Für letzteres möchte ich die recht bekannten Beispiele aus dem Gebiet des Kalenderwesens erwähnen. Als für das Jahr 1700 in den protestantischen deutschen Ländern die Kalenderreform eingeführt wurde, erteilte der brandenburgische Kurfürst das Privileg für seinen Herrschaftsbereich der Berliner Akademie, der sächsische Kurfürst dem Leipziger Drucker Thomas Fritzsch und in Hessen gab es ein landgräfliches Privileg für einen mehrfach wechselnden Drucker. In der Regel suchte man sich dann einen zuverlässigen Kalenderautor, so in Berlin Gottfried und später Christfried Kirch, oder in Kassel Matthias Weete. ${ }^{2}$

Privilegien wurden also nicht nur vom Kaiser gewährt, sondern ebenso von anderen Fürsten, dann natürlich nur für ein bestimmtes Territorium geltend.

Zurück nach Wien und zu den dortigen Privilegien. Worin besteht ihre Bedeutung für die Astronomiegeschichte? - wobei das folgende natürlich auch für andere Bereiche der Geschichtsforschung gilt. Zunächst geben die Privilegien in vielen Fällen Aufschluß über das Werden eines Werkes, auch im Zusammenhang mit den gelegentlichen Ausführungen, die über das geplante Werk vom Autor gemacht werden, dazu von mir das Beispiel Jost Bürgi. Der andere Fall ist, daß wir Einblicke in das geplante Lebenswerk eines Autors bekommen, das zu verwirklichen dem Autor aus unterschiedlichen Gründen nicht vergönnt war - sei es durch seinen plötzlichen Tod, dazu das Beispiel Erasmus Reinhold, oder eine anderweitige Störung der wissenschaftlichen Arbeit - das am Beispiel Christoph Rothmanns.

\section{Beispiel: Jost Bürgi}

Nehmen wir die Geschichte des Buches von Jost Bürgi (1552-1632) über das sogenannte Triangularinstrument. Ein erstes Ersuchen für die Erteilung eines

\footnotetext{
${ }^{1}$ s. dazu Schneider, Ivo: Urheberrechtliche Sicherung im naturwissenschaftlichen Schrifttum des 16. Jahrhunderts. Buchprivilegien bei Gemma Frisius (1508-1555). In: Börsenblatt für den Deutschen Buchhandel / Frankfurter Ausg. 30 (1974), S. A 145-151

${ }^{2}$ Herbst, Klaus-Dieter: Die Kalender von Gottfried Kirch. In: Beiträge zur Astronomiegeschichte, Band 7. Frankfurt a. M. 2004 (Acta Historica Astronomiae; 23), S. 115-159; Hamel, Jürgen: Die Kalenderreform des Jahres 1700 und ihre Durchsetzung in Hessen. In: Zeitschrift des Vereins für hessische Geschichte und Landeskunde 105 (2000), S. 59-74
} 
Privilegs sowohl für das Instrument, als auch für das dieses beschreibende Buch ist aus dem Jahre 1602 bekannt.

Interessanterweise stammt dieses Schreiben nicht von Bürgi selbst, sondern vom Erzherzog Maximilian, dem Bruder des Kaisers, der sich für Bürgi einsetzt. $\mathrm{Da} B$ Bürgi in Beziehung zum Erzherzog trat, war bislang nicht bekannt, muß aber der Fall gewesen sein, weil es nicht denkbar ist, daß Maximilian bei seinem Bruder ohne genügende Sicherheiten Fürsprache hielt. Sicherlich geschah dies während Bürgis Aufenthalt in Prag. Mit Bezug auf dieses Schreiben ersucht Bürgi 1611 noch einmal persönlich um die Erteilung des Privilegs, wobei nicht ganz klar wird, ob es um eine Verlängerung geht, oder das Privileg 1602 nicht erteilt wurde. Wie auch immer, das Buch erschien ohnehin erst nach weiteren 37 Jahren, 1648 nach dem Tod des Autors durch Bürgis Schwager Benjamin Bramer herausgegeben ${ }^{3}$. Die Umstände, die zu diesem Zeitablauf führten, können hier nicht erörtert werden. Es dürfte jedenfalls auch damit zu tun haben, daß der geniale Bürgi wegen seines Mangels an formaler akademischer Bildung, darunter des Mangels an Kenntnissen der lateinischen Sprache, eine Scheu vor dem Publizieren hatte. Für diesmal reicht die Erkenntnis, daß durch die Analyse der Privilegien und nur auf diesem Weg ein Rückschluß auf die Entstehungsgeschichte des Werkes Bürgis möglich ist, auf die Zeit, die Bürgi für seine Arbeit benötigte, um sie privilegreif zu entwickeln - und dann doch unvollendet ließ.

Erhalten sind weiterhin die Vorgänge zu Bürgis Buch über die Erfindung der Logarithmen ${ }^{4}$, die Ersuchung um ein Privileg für den "weilandt Kayßer Rudolphi undt Matthiae christmildester gedachtniß gewester Cammer Uhrmacher Jobst Bürgy".

\section{Beispiel: Erasmus Reinhold}

Erasmus Reinhold (1511 - 1553) gehörte in Wittenberg zum Gelehrtenkreis um Philipp Melanchthon und übernahm 1536 die dortige Professur für höhere Mathematik, welche die Astronomie einschloß. Übrigens erhielt kurz darauf Georg Joachim Rheticus die Professur für niedere Mathematik, d.h. Geometrie und Arithmetik. Beide waren also Kollegen und es ist klar, daß auch Reinhold in die frühen Diskussionen um das neue Weltsystem eingebunden war. Diese führten schließlich auf der einen Seite zur Reise des jungen Rheticus zum alternden Copernicus mit den bekannten Folgen für die Herausgabe des berühmten Werkes, andererseits durch Reinhold zur Bearbeitung der "Prutenischen Tafeln", zu Planetentafeln unter Verwendung copernicanischer Daten, die für die Rezeption des heliozentrischen Weltsystems von großer Bedeutung wurden.

\footnotetext{
${ }^{3}$ Bramer, Benjamin: Apollonius cattus oder kern der ganzen geometriae. Kassel 1648

${ }^{4}$ Bürgi, Jost: Arithmetische und geometrische Progress Tabulen. Prag 1620
} 
Das Privileg liegt im Entwurf eines Schreibers vor, datiert Prag, den 24. Juni 1549 im Umfang von vier Folioseiten. Die Schrift ist sehr sauber, es gibt nur an zwei Stellen überschriebene Worte, wohl vom Schreiber selbst sowie an drei Stellen Korrekturen eines Wortes, eine am Rand notierte Ergänzung sowie an Ende einen Schlußsatz mit der Datierung (ein zuvor begonnener Satz des Schreibers wurde dafür ausgestrichen) - all dies von anderer Hand.

Die "Prutenischen Tafeln" stellte Reinhold an die Spitze (Nr. 1) seiner Liste, für die er um ein Privileg, ein "Diplom" (so im letzten getilgten Satz) nachsuchte.

Wie aber ging es mit den insgesamt 16 dort aufgelisteten Werken weiter? Außer den "Prutenischen Tafeln" erschienen nur noch drei Bücher im Druck, die "Tabulae directionum", posthum 1554 (Nr. 3), Ephemeriden für die Jahre 1550 und 1551 (Nr. 4), sowie die evtl. unter Nr. 10 gemeinte Herausgabe der "Theoricae novae planetarum" Peuerbachs von 1542. Der Kommentar zu Copernicus (Nr. 13) blieb im Manuskript von Reinholds Hand erhalten und wurde erstmals 2002 ediert $^{5}$. Der Vollständigkeit halber sei erwähnt, daß einige kleinere Texte Reinholds, eher Gelegenheitsschriftchen, größeren Werken beigedruckt wurden ${ }^{6}$, die hier außer acht gelassen werden können und auch im Privilegantrag nicht erwähnt werden.

Von allen anderen Vorhaben ist weder etwas gedruckt worden, noch ist sonst anderweitig etwas bekannt. Wie kam es dazu? Der Grund ist ein recht tragischer. Der vielversprechende, erst 42jährige Gelehrte, verstarb 1553, zwei Jahre nach Erscheinen der Erstauflage seiner berühmten Tafeln, in seiner Heimatstadt Saalfeld in Thüringen an der Pest. Sein anspruchsvolles Publikationsprogramm blieb ein Fragment.

Reinholds Publikationsprogramm nach dem Text des Privilegs (die gedruckten Werke kursiv)

[1] Nouae tabulae Astronomicae forma Alphonsina et Copernici, quae exhibent emendatum calculum motuum coelestium omnium congruentem cum obseruationibus tum priscis, tum recentibus. Id quod nec ptolemaicae tabulae praestant, nec Alphonsinae, nec ullae ex ijs propagatae. Prutenicae tabulae coelestium motuum. Tübingen 1551 (weitere Ausgaben 1562, 1571, 1585)

[2] Tabulae resolutae ex prioribus deriuatae, ex quibus facilima sit supputio motuum coelestium. His insertae sicut tabulae Eclipsium, quae suppeditant uerum calculum omnium deliquiorum Solis et lunae retro ad tria millia annorum.

[3] Tabularum directionum, ut uocant, generalium primus liber cum secundo libro particularium tabularum. Primus liber tabularum directionum discentibus prima elementa astronomiae necessarius $\&$ utilissimus. Tübingen 1554

\footnotetext{
${ }^{5}$ vgl. Anmerkung zu Nr. 13 des Privilegs

${ }^{6}$ Verzeichnis der im deutschen Sprachbereich erschienenen Drucke des XVI. Jahrhunderts. VD 16, 2. Abt., Band 2. Stuttgart 1997
} 
[4] Ephemerides singulorum annorum aliquot futurorum calculatae ex his recentibus tabulis. Ephemerides duorum annorum 50. et 51. Tübingen 1550

[5] Tabulae ortuum et occasuum plurimarum stellarum fixarum, tum ad ueterum uaria tempora, tum ad nostra per multa climata.

[6] Chronicon, in quo priori pagina non solum annorum series deducta est a uarijs initijs, quae firmissimis rationibus constituta sunt, uerum et Eclipses luminum ad singulos annos, Loca trium superiorum planetarum, ex magni congressus planetarum, item Meteora, quae passim in historijs annotata sunt. Reliqua pagina regione habet historica tanquam effectus causarum caelestium distributa in quatuor classes, uidelicet in physica, seu Oeconomica, philosophica, politica et Ecclesiastica.

[7] Calendarium Ecclesiasticum quod continet ex ipsis fontibus deductam doctrinam eam de anno et mensibus, quae traditur in computo Ecclesiastico, quod aeditum quidem est sed augebitur.

[8] De historia annorum seu Calendarium Astronomicum profuturum doctis, ni quo inter cetera illustris est tractatio de anno Aegiptico et Graeco una cum nouis tabulis et eruditis, siue quibus ptolemaei magna constructio seu almagestum, et similia scripta difficilime intelligunt.

[9] Isagoge sphaerica, seu doctrinae primi mobilis elementa quinque libris comprehensa.

[10] Hypotyposes orbium coelestium, quas uulgo uocant Theoricas planetarum, congruentes cum tabulis Astronomicis supradictis.

[evtl.]: Theoricae novae planetarum Georgii Purbachii Germani ab Erasmo Reinholdo Salueldensi pluribus figuris auctae. Wittenberg 1542

[11] Compositio noua Quadrantis cum multis utilissimis tabulis.

[12] Doctrina triangulorum planorum et sphaericorum ea methodo, quae scholarum usui accomodata est, cum secundo canone per singula scrupula extenso, quem licet omnium astronomicarum tabularum fundamentum adpellare.

[13] Eruditus commentarius in totum opus Reuolutionum Nicolai Copernici.

[Handschrift] Commentarius in opus revolutionum Copernici; Staatsbibliothek Berlin, Preußischer Kulturbesitz, Ms. lat. fol. 391

Edition und zugl. erste Veröffentlichung in: Nicolaus Copernicus Gesamtausgabe, Band VIII.1. Berlin 2002

[14] Geometrica uaria, inter quae circuli quadratura, ac Erudita confutatio ... Item Commentarius in Quintum et Decimum librum Euclidis.

[15] Commentarius in Geographiam ptolomaej cum noua uersione latina.

[16] Optice Arabis alhazen hactenus non edita, correcta et figuris utiliter illustrata. 


\section{Beispiel: Christoph Rothmann}

Neben den nächtlichen Arbeiten auf der Sternwarte des Landgrafen Wilhelms IV. von Hessen in Kassel hatte sein Astronom Christoph Rothmann ein umfangreiches Programm astronomischer Arbeiten konzipiert. Für dieses bekam er am 6. Mai 1588 von Rudolf II. ein kaiserliches Privileg auf $20 \mathrm{Jahre}^{7}$. Die kaiserliche Archivfassung hat sich, ausgefertigt von Jacob Curtius, in der Wiener Sammlung der Impressorien erhalten, das Original und der eigenhändige Entwurf Rothmanns befinden sich in Marburg bzw. Kassel ${ }^{8}$. Es handelt sich, wie auch bei Reinhold um ein Autor-Spezialprivileg, in dem, wie zu erwarten ist, nur der Autor und kein bereits avisierter Drucker begünstigt wird.

Rothmanns Publikationsprogramm nach dem Text des Privilegs (die erhaltenen Werke kursiv)

[1] Observationes stellarum fixarum institutae Casselis sumptibus et instrumentis Illmi Principis Guilielmi Landgrauij Hassiae ec ...; vna cum noua theorica praecessionis aequinoctiorum et mutatae obliquitatis Zodiaci ex his obseruationibus eruta. Christophori Rothmanni Bernburgensis, illustrissimi principis Guilielmi, Landgravii Hassiae etc. Mathematici, observationum stellarum fixarum liber primus; Kassel UB, $2^{\circ} \mathrm{Ms}$. astron. 5 [7, $85 \mathrm{Bl}$. Edition und zugl. erste Veröffentlichung in: Christoph Rothmanns Handbuch der Astronomie von 1589. Kommentierte Edition der Handschrift Christoph Rothmanns "Observationum stellarum fixarum liber primus", Kassel 1589. Hrsg. und komm. von Miguel A. Granada, Jürgen Hamel und Ludolf v. Mackensen. 2003 (Acta Historica Astronomiae; 19)

[2] Dialexis cometae, qui anno Christi MDLXXXV. mensibus Octobri et Nouembri apparuit, in qua demonstratur, quod cometae non sint FLNF,4H vicinarum stellarum, neque corpora cum reliquis stellis perpetua neque halitus in aere accensi, sed quod sint corpora temporanea.

Snellius: Willebrord: Descriptio cometae, qui anno 1618 mense Novembri primum effulsit. Huc accessit Christophori Rhotmanni Ill. Princ. Wilhelmi Hassiae Lantgravii Mathematici descriptio accurata cometae 1585. Leiden 1619

\footnotetext{
${ }^{7}$ Druck des vollständigen Textes in Miguel A. Granada: Sfere solide e cielo fluido. Momenti del dibattito cosmologico nella seconda met del Cinquecento. Milano 2002 (Istituto Italiano per gli studi Filosofici / Saggi; 41), App. 2; die enthaltenen Werke und ihre Erhaltungsformen in Jürgen Hamel: Die astronomischen Forschungen in Kassel unter Wilhelm IV. Mit einer Teiledition der deutschen Übersetzung des Hauptwerkes von Copernicus um 1586. Thun; Frankfurt 1998, 2. korrigierte Aufl. 2002 (Acta Historica Astronomiae; 2), S. $77 f$.

${ }^{8}$ Hessisches Staatsarchiv Marburg 4a, 31-17 bzw. Universitätsbibliothek Kassel, HSA $2^{\circ}$ Ms. astron. $5[11$
} 
[3] Organon mathematicum, constans logistica sexagenaris. Doctrina sinuum, doctrina de compositione rationum, seu ut vulgo vocatur regula sex quantitatum et doctrina triangulorum, aureis compendiis referta, quibus omnia, tam in sphaericis, quam planis triangulis solo additionis et subductionis calculo facilime indagantur, quo cognito quilibet suo Marte Ptolomaeum, Copernicum, Regiomontanum reliquosque artifices facilime intelligere, canones astronomicos vt ascensionum, prostaphaereseon, latitudinum, parallaxium ec. ipse condere, aut etiam sine canonibus astronomicis motus planetarum subducere, et de meteoroscopica, geodesia, optica, catoptrica, gnomonica, geographia, astronomia deque omnibus doctrinis, quae e geometriae et arithmeticae fontibus promanant, recte et dextere judicare potest.

Organon mathematicum, contens logistica sexagenaria, doctrina sinuum, et doctrina triangulorum. Quo cognito quilibet suo marte Ptolemaeum, Copernicum, Regiomontanum, reliquosque artificos facilime intelligere: Canones astronomicos, ut ascensionum, prosthaphaereseon, latitudinum, parallaxium etc. ipse contere, aut etiam sine canonibus astronomicis motus planetarum subducere: et de meteoroscopica, geodesia, optica, dioptrica, gnomonica, geographia, astronomia, deque omnibus, doctrinis quae e geometriae et arithmetricae fontibus promanant, recte et dextere iudicare potest; Kassel UB, $4^{\circ} \mathrm{Ms}$. math. 29, $255 \mathrm{Bl}$.

[4] Elementa astronomica, in quibus hypotheses Ptolomaicae ex hypothesibus Copernici corriguntur et supplentur ipsoque vsu et calculo nouo tabularum Tychonicarum seu Danicarum declarantur. Astronomia: In qua hypotheses Ptolemaicae ex hypothesibus Copernici corriguntur et supplentur: et inprimis intellectus et usus tabularum Prutenicarum declaratur et demonstratur; Kassel UB, $4^{\circ}$ Ms. astron. 11, $183 \mathrm{BI}$.

[5] Astronomia noua ex nouis et accuratissimis obseruationibus, cum veteribus collatis, restituta, in qua nouo et nobilissimo compendio omnes planetarum motus luminarumque eclypses solo additionis et subductionis calculo absque ullis tabulis astronomicis, ad quoduis tempus, facilime indagantur.

[6] Scholae astronomicae, seu commentarius in totum opus reuolutionum Copernici nouis obseruationibus refertus.

[7] Tractatus de parallaxibus earumque obseruatione.

[8] Optica breuis et perspicua, in artis formam redacta.

[9] Regula coss, seu algebraica, logica methodo tractata.

[10] Doctrina de meteoris, opticis et physicis fundamentis innixa.

[11] Astrologia juditiaria facilis ab ethnicis et impiis futilitatibus repurgata, solisque physicis causis innixa.

[12] Scholae astrologicae, in quibus omnium praeceptionum

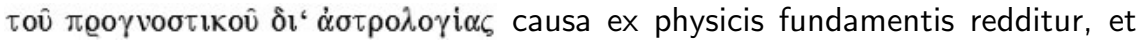
reliquae ethnicae et impiae futilitates solide refutantur. 
Betrachtet man die konzipierten Werke nach inhaltlichen Gesichtspunkten, wird man gewahr, daß Rothmann ein komplettes Programm der Astronomie entworfen hat, mit allen Nebengebieten der Astrologie und der Mathematik / Trigonometrie, ausgenommen den Kalender und darin auch seine Anerkennung des copernicanischen Weltsystems darin zum Ausdruck kommt. Rothmanns Programm stellte kein abstraktes Vorhaben weit jenseits der Realität dar. Denn für mehrere Arbeiten existieren fertige oder weitgehend abgeschlossene Manuskripte. Sie beweisen, daß Rothmann in der Verwirklichung seiner privilegierten Vorhaben weit gediehen war und die Fähigkeit gehabt hätte, diese Arbeiten zuende zu führen.

Hätte Rothmann das durch Rudolfs Privileg geschützte Publikationsprogramm ausgeführt, dann - so darf freilich etwas spekulativ geurteilt werden - wäre Christoph Rothmann in die Geschichte als einer der bedeutendsten Astronomen des späten 16. Jahrhunderts eingegangen, in einer Reihe mit Michael Mästlin und Tycho Brahe stehend. Doch es kam anders, publiziert wurde lediglich Rothmanns Arbeit über Kometen (Nr. 2), von höchster Bedeutung nicht nur für die Kometenforschung, aber auch dies erst 1619. Sein Sternkatalog erschien erstmals $1666^{9}$. Der Kommentar zum Katalog, "Observationum stellarum fixarum liber primus" (Nr. 1), enthaltend weite Abhandlungen zur theoretischen Astronomie, einschl. der Instrumentenkunde, wurde erstmals 2002 ediert. Details können hier nicht weiter verfolgt werden (vgl. die in Anm. 7 und 9 genannten Arbeiten), nur kurz die Frage, warum unterblieb die Veröffentlichung?

Nachdem Rothmann seinen Sternkatalog abgeschlossen und seinem Auftraggeber und Landesherren Landgraf Wilhelm in einer erhaltenen repräsentativen Pergamenthandschrift übergeben hatte ${ }^{10}$, nahm seine Lebensgeschichte einen Verlauf, der alle weiteren Pläne unerledigt ließ. Im Mai 1590 trat Rothmann eine Reise zu Tycho Brahe an, wo er dessen Instrumente studieren und in einen wissenschaftlichen Gedankenaustausch treten sollte. Brahe hatte Rothmann in Kopenhagen empfangen und traf mit ihm am 1. August 1590 auf der Insel Hven ein, wo er bis zum 1. September blieb und die Insel wieder verließ.

Unter Bruch aller rechtlichen Verpflichtungen und sonstiger Gewohnheiten kehrte Rothmann aber nie mehr nach Kassel zurück. Das Ausbleiben Rothmanns ist mehrfach Gegenstand des Briefwechsels zwischen Wilhelm und Brahe, doch alle Hoffnungen Wilhelms, daß Rothmann seine Forschungen in Kassel weiterführen würde, blieben vergeblich.

\footnotetext{
${ }^{9}$ Eine Untersuchung des Sternkatalogs mit Ableitung der statistischen Fehler der darin verzeichneten Sternpositionen erfolgte in Hamel, Die astronomischen Forschungen (wie Anm. 7, S.\#). Die darin enthaltenen Sternörter waren nach Berechnungen von E. Rothenberg (Berlin) mit einem deutlich kleineren statistischen Fehler behaftet, als die des Katalogs von Brahe!

${ }^{10}$ Universitätsbibliothek Kassel, HSA $2^{\circ}$ Ms. astron. 7
} 
Von nun mündet Rothmanns Lebensweg in ein uns nicht recht verständliches Trauerspiel, auch zum erheblichen Nachteil der astronomischen Forschung. Anstatt sich an seinen Arbeitsort Kassel zu begeben, begab er sich in seine Heimatstadt Bernburg im Anhaltischen. Welcher Tätigkeit er dort nachging wissen wir nicht, er verfaßte noch eine Arbeit über das Sakrament der Taufe und das sind dann alle Informationen, die wir von ihm haben. Auch sein Todesdatum ist unbekannt.

In Kassel ließ Rothmann alle seine fertigen oder weitgehend abgeschlossenen, in umfangreichen Manuskripten vorliegenden Werke zurück. Das erhaltene Privileg zeigt uns, wie zielstrebig Rothmann seine Forschungen verfolgte und welche Pläne er weiterhin, über das Erhaltene hinausgehen hatte - bis sein Leben einen anderen Verlauf nahm. ${ }^{11}$

\section{Eine Übersicht}

Nach einer kurzen und unvollständigen Durchsicht des Registers der Impressorien finden sich Unterlagen zu folgenden Astronomen und Druckern oder Verlegern mit astronomischer Relevanz:

Philipp Apian 1582, Caspar Bartholinus 1623, Wilhelm und Johannes Blaeu 1678 bzw. 1621, Tycho Brahe 1586 und 1590, Georg Caesius für Kalender und Prognostiken, Sethus Calvisius 1610 und 1651, Nathan Chytraeus 1575, Nicodemus Frischlin, Thaddaeus Hagecius, Georg Hanschius 1718 für die Herausgabe der Kepler-Werke, Johannes Kepler 1617 und 1627 (29?), David Herlitzius, Johannes Hevelius 1662, Levinus Hulsius, ? Hofmann für Kalender, Johann Krabbe 1607, Stanislaus Lubienietzki 1666, Michael Mästlin 1580, Albin Moller, Caspar Peucer 1569-1570, Andreas Reyher, Nicolaus Raimarus Ursus, Julius Schiller, Christoph Schißler, Tilemann Stella, Matthias Wasmuth sowie Erhard Weigel und das "Collegium consultorum".

Drucker und Verleger mit bedeutenden astronomischen Büchern: Arnold Birckmann 1559, Verlagshaus Elzevier, Familie Endter 1659-1704, Felsecker 1700 -1743, Thomas Fritzsch, Sebastian Henricpeter (Verlagsprogramm mit astronomischen Werken), Peter Perna, Familie Wechel.

Eine systematische Durchsicht würde ganz sicher weiteres erschließen. Die Nutzung dieser Quelle der Wissenschaftgeschichte könnte uns manchen Aufschluß geben. Eine Publikation und inhaltliche Analyse weiterer Privilegschriften befindet sich in Vorbereitung.

\footnotetext{
${ }^{11}$ vgl. zur Biographie Rothmanns vgl. Hamel: Die astronomischen Forschungen, 14-19, 77-84 sowie Christoph Rothmanns Handbuch der Astronomie von 1589. Kommentierte Edition der Handschrift Christoph Rothmanns "Observationum stellarum fixarum liber primus", Kassel 1589. Hrsg. und komm. von Miguel A. Granada, Jürgen Hamel und Ludolf v. Mackensen. 2003 (Acta Historica Astronomiae; 19), S. 10-19, 32-48
} 

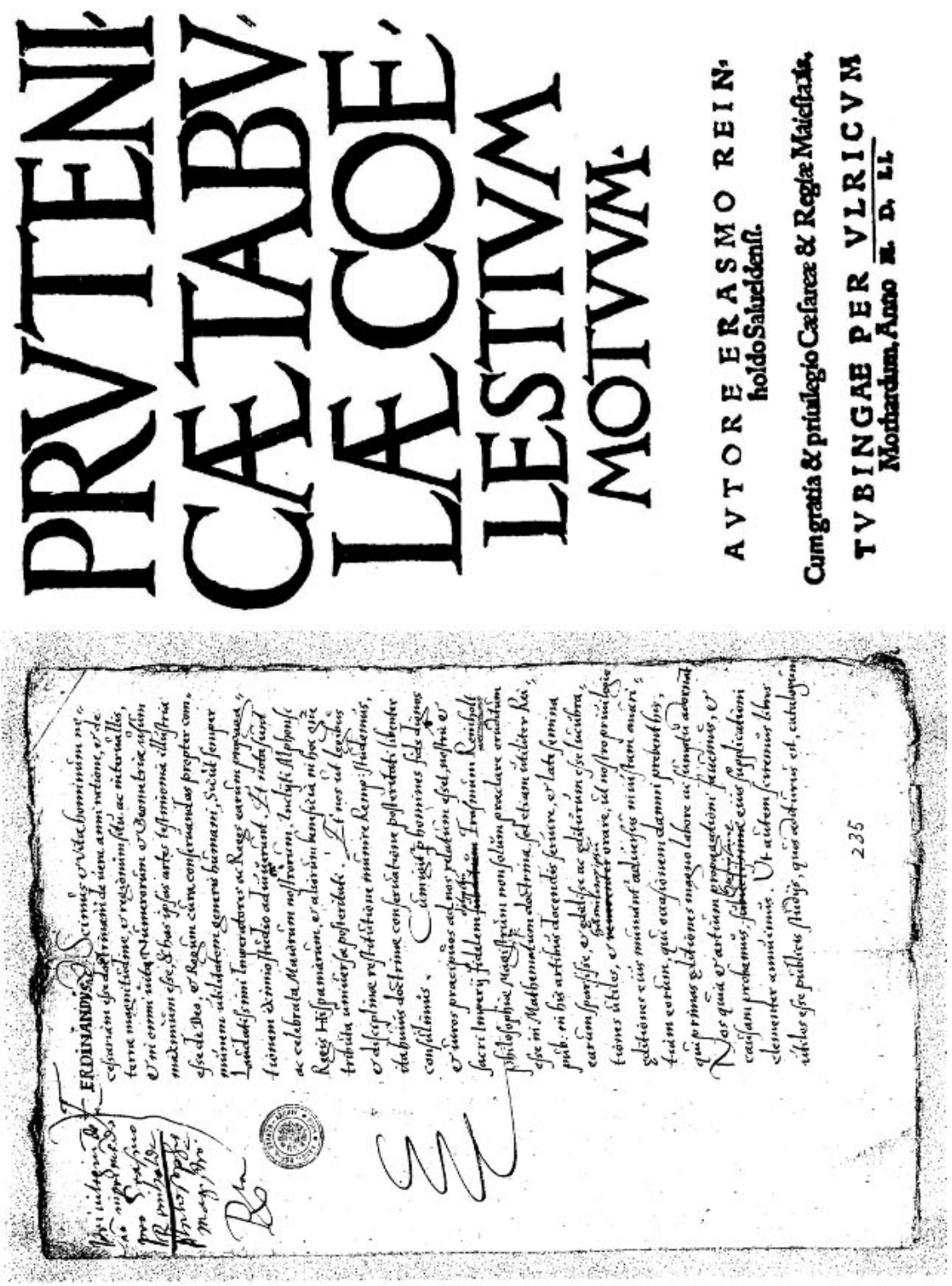

Abb. 1: unten: Erste Seite des Privilegs für Erasmus Reinhold (Österr. Staatsarchiv Wien, Haus-, Hof- und Staatsarchiv, Bestand Impressorien, Reinhold/oben: Titelseite der "Prutenischen Tafeln" von Erasmus Reinhold, Tübingen 1551 mit dem Vermerk des kaiserlichen Privilegs 